\title{
S-苯并噻吩盐的合成的方法及其反应的研究进展
}

\author{
张变香* 常 姣杨 祺 吴 群 \\ (山西大学化学化工学院 太原 030006)
}

\begin{abstract}
摘要 综述了近年来合成苯并噻吩盐的多种方法, 讨论了其存在的优缺点, 并详细介绍了 $S$-苯基苯并噻吩盐的 $[4+2]$ Diels-Alder 反应、加热反应、光解反应以及开环反应，并对其发展前景做出了展望.
\end{abstract}

关键词 $S$-苯并噻吩盐; 加热反应; 光解反应; 开环反应; [4+2] Diels-Alder 反应

\section{Progress in Synthesis and Reaction of S-Benzothiophenium Salts}

\author{
Zhang, Bianxiang* Chang, Jiao Yang, Qi Wu, Qun \\ (School of Chemistry and Chemical Engineering, Shanxi University, Taiyuan 030006, China)
}

\begin{abstract}
The synthetic methods of $S$-benzothiophenium salts is reviewed and the advantages and disadvantages of various methods are discussed. The $[4+2]$ Diels-Alder reaction, thermolysis, photolysis, ring-opening reaction of $S$-phenylbenzothiophenium salts are introduced in details. And the development of the chemistry and applications of $S$-phenylbenzothiophenium salts is also prospected.
\end{abstract}

Keywords $S$-benzothiophene salt; [4+2] Diels-Alder reaction; thermolysis; photolysis; ring-opening reaction

苯并噻吩及其衍生物是自然界中存在的含硫杂原 子的环状化合物之一, 不仅是重要的有机合成中间体, 而且作为医药、农药、功能性材料等的基本骨架被广泛 利用 ${ }^{[13]}$. 苯并噻吩通常指苯并 $[b]$ 噻吩, 许多含有苯并 噻吩结构的化合物都具有消炎镇痛的作用, 而且在医药 合成上带有噻吩环的抗生素比苯基同系物具有更好的 疗效 ${ }^{[4 \sim 9]}$. 苯并噻吩常用来合成一些目标产物的中间体, 但因其特殊芳香性结构, 反应的转化率较低 ${ }^{[10]}$. 当噻吩 环上的硫原子被氧化后, 其反应活性明显提高, 可以作 为双烯体系有效地进行 $[4+2]$ 环加成反应 ${ }^{[11,12]}$. 苯并噻 吩及其衍生物的研究在我国还处在起步阶段, 相关试剂 及药物价格比较昂贵, 对其化合物的合成及其性质进行 讨论、研究具有十分重要的意义. 三芳基锍鎓盐是一类 重要的有机硫化合物, 它可用作阳离子光引发剂、相转 移催化剂、光生酸剂、阳离子荧光探针以及生物传感器 等，在有机合成上锍鈆盐可以进行多种类型的反 应 $^{[13 \sim 15]}$. 因此, 以苯并噻吩为骨架的锍鎓盐的合成及 性质研究受到了广泛关注. 本文将结合自己的研究较详
细地介绍苯并噻吩盐的几种合成方法以及其有趣的结 构性质和反应特征(Scheme 1).<smiles>[R]c1sc2ccccc2c1[R]</smiles><smiles>[R][SH]([R])[R]</smiles><smiles>[R]c1c([R])[s+](-c2ccccc2)c2ccccc12</smiles>

Scheme 1

\section{1 苯并噻吩盐的合成}

\section{$1.1 S$-烷基苯并噻吩盐的合成}

1969 年, Acheson 等 ${ }^{[16 ~ 20]}$ 的研究发现 $\mathrm{AgBF}_{4}$ 是一种 对硫进行烷基化的较好催化剂, 在它的作用下, 苯并噻 吩及其衍生物与碘代甲烷反应可以得到产率较好的 $S$ 烷 基苯并噻吩盐 $\mathbf{1}$ (Eq. 1).

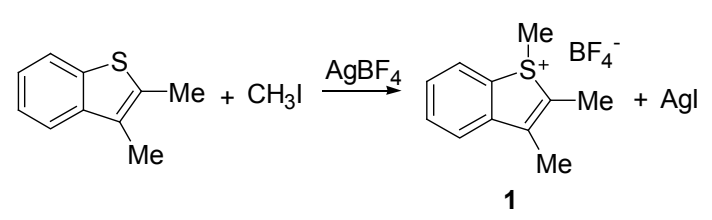

*E-mail: zbxthh@sxu.edu.cn

Received October 27, 2011; revised January 10, 2012; published online February 4, 2012.

Project supported by the Shanxi Science and Technology Department (No. 20110321040-02) and the Shanxi Scholarship Council of China (No. 2010-001). 山西省科技攻关项目(No. 20110321040-02)和山西省回国留学人员科研(No. 2010-001)资助项目. 
$S$-烷基苯并噻吩盐在亲核性溶剂中不稳定, 很容易 脱去烷基回到苯并噻吩反应底物. 为了更好地研究苯并 噻吩盐的化学性质, 稳定的 $\mathrm{S}-\mathrm{C}$ 键的形成成为研究的 热点. 1986 年, Kitamura 等 ${ }^{[21 a]}$ 利用邻位有芳硫基的取代 苯乙烯合成了 $S$-苯基苯并噻吩盐 2 (Eq. 2). 由于 $\mathrm{S}-\mathrm{Ph}$ 键较 $\mathrm{S}$ - $\mathrm{Me}$ 稳定, $S$-苯基苯并噻吩盐在极性溶剂中可以 稳定存在, 如甲醇和 DMSO 等, 这为研究苯并噻吩盐 的化学性质提供了保障.

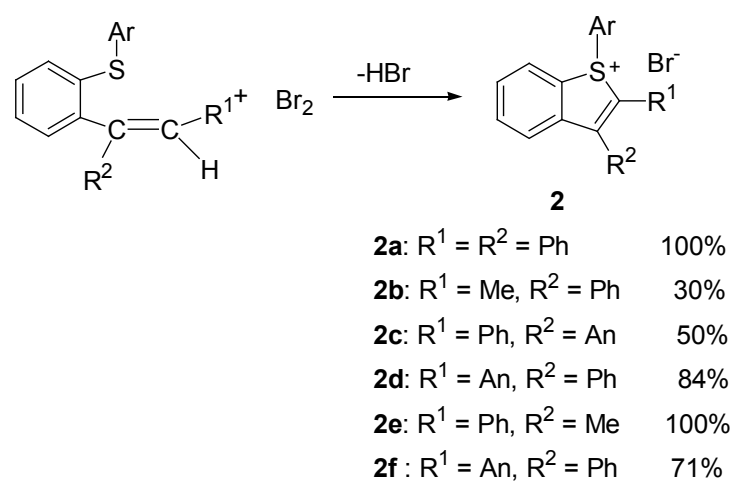

在这个反应体系中, $S$-芳基苯并噻吩盐生成的容易 程度，与取代基溶剂的极性有关. $\mathrm{R}^{1}$ 为芳基 $\mathrm{R}^{2}$ 为烷基时, 溶剂的影响效果以 $\mathrm{AcOH}>\mathrm{CH}_{2} \mathrm{Cl}_{2}>\mathrm{CCl}_{4}$ 的顺序递减, 极性溶液 $\mathrm{AcOH}$ 中因存在游离的离子对, 目标产物的产 率较高, 有利于分子内环化 ${ }^{[21]}$.

同样，与以上取代苯乙烯相对应, 1989 年, Kitamura 等 ${ }^{[22]}$ 用 2 -硫芳基-苯乙炔的分子内环化合成了 $S$-苯基苯 并噻吩盐. 在 $\mathrm{C} \equiv \mathrm{C}$ 的亲电反应中, 经过了桥架正离子 的分子内环化反应, 生成了硫盐 3 (Scheme 2). 在这个 体系中, 反应条件温和, 而且可以通过亲电试剂导入官 能性基团. 当 $\mathrm{R}^{1}$ 为苯基或对甲氧基苯基时, 桥架的中间 体由于有硫原子的存在, 容易发生分子内环化生成稳定 的苯并噻吩盐.

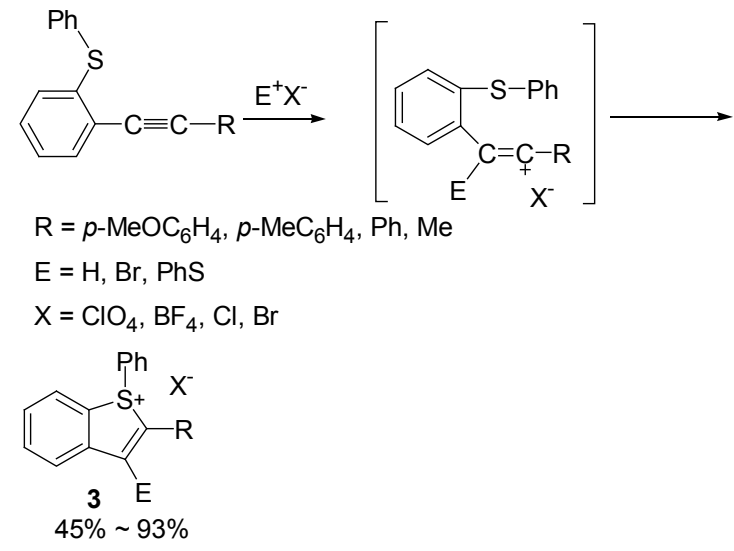

\section{Scheme 2}

在以上两个反应中, 亲电诱导环化合成 $S$-苯基苯并
噻吩盐的反应底物较难合成, 需要多步反应, 产率和原 子经济性都不高, 并且还必须满足中间正离子产物能够 稳定地存在. 这两种方法不能用于无取代基的 $S$-苯基苯 并噻吩盐的合成.

1997 年, Kitamura 等 ${ }^{[23]}$ 用苯并噻吩与二苯基高碘盐 反应，成功地合成了在有机合成上被认为是难以合成的 无取代 $S$-苯基苯并噻吩盐. 在 $1 \mathrm{~mol} \%$ 醋酸铜催化下, 苯 并噻吩与 1.2 equiv. 的二苯基碘三氟甲磺酸盐, $140{ }^{\circ} \mathrm{C}$ 下, 反应 $30 \mathrm{~min}$ 后，可以得到较高产率的 $S$-苯基苯并噻吩盐 (Eq. 3). 这种方法非常简单实用, 弥补了亲电诱导环化 合成 $S$-苯基苯并噻吩盐的不足. 当 C-2 和 C-3 位都有苯 基或对甲氧基苯基时，目标化合物 $5 \mathbf{i}$ 和 $5 \mathbf{j}$ 的产率明显 降低，说明了两个苯基和对甲氧基苯基对硫的苯基化有 空间位阻的影响.

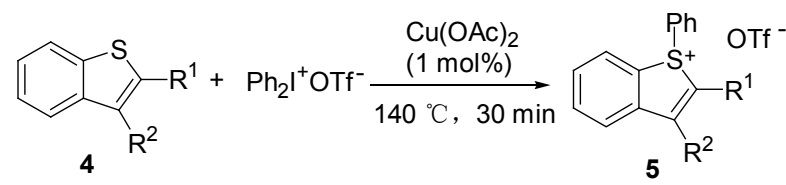

a: $R^{1}=R^{2}=H \quad 83 \%$

b: $R^{1}=M e, R^{2}=H \quad 94 \%$

f: $R^{1}=M e, R^{2}=P h \quad 94 \%$

c: $R^{1}=H, R^{2}=$ Me $81 \%$

g: $R^{1}=P h, R^{2}=M e \quad 88 \%$

d: $R^{1}=P h, R^{2}=H \quad 93 \%$

h: $R^{1}=R^{2}=M e$

$94 \%$

e: $R^{1}=H, R^{2}=P h \quad 70 \%$

i: $\mathrm{R}^{1}=\mathrm{R}^{2}=\mathrm{Ph} \quad 69 \%$

j: $\mathrm{R}^{1}=\mathrm{R}^{2}=p-\mathrm{MeOC}_{6} \mathrm{H}_{4} \quad 42 \%$

铜(II)催化苯并噻吩苯基化反应的可能机理如 Scheme 3 所示. 早年 Beringer ${ }^{[24]}$ 和 Caserio 等 ${ }^{[25]}$ 报道了 二价铜催化二芳基碘鎓盐的分解反应，在反应机理中讨 论了在甲醇、水等溶剂中 $\mathrm{Cu}(\mathrm{II})$ 被还原成 $\mathrm{Cu}(\mathrm{I})$. 同样, 1983 年 Lockhart 等 ${ }^{[26]}$ 报道了 $\mathrm{Cu}(\mathrm{I})$ 是催化二芳基碘鎓盐 分解反应的原因, 2008 年 Phipps 等 ${ }^{[27]}$ 详细讨论了 $\mathrm{Cu}(\mathrm{II})$ 催化吲哚的芳基化反应. 由此, 可以推测在苯并噻吩苯 基化反应机理中, 首先 $\mathrm{Cu}(\mathrm{II})$ 在苯并噻吩的作用下被还 原成 $\mathrm{Cu}(\mathrm{I})$, 一价铜与二芳基碘鎓盐结合生成 $\mathrm{PhCu}(\mathrm{III})$ 中间体, 然后苯基铜(III)中间体进攻硫原子, 生成了 $S$ 苯基苯并噻吩盐和有催化活性的 $\mathrm{Cu}(\mathrm{I})$.

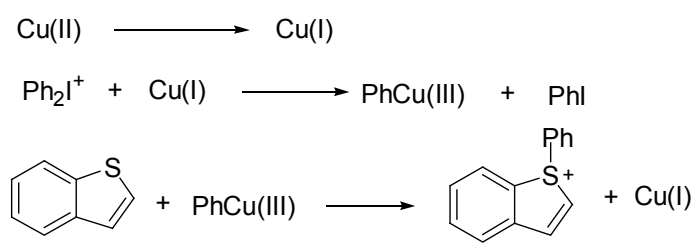

Scheme 3

\section{$2 S$-苯基苯并噻吩的晶体结构紫外光谱分析}

$S$-苯基苯并噻吩盐 (5a)的构造和对应的苯并噻吩不 同，由其 $X$ 射线单晶衍射(图 1)测试分析可以得出以下 
几点很有趣的特征: (1)三氟甲磺酸根负离子 (OTf $)$ 的氧 原子和硫原子的距离小于氧原子和硫原子的范德华半 径之和, 存在较强的相互作用力. (2)硫原子周围是四面 体结构, 属于 $\mathrm{sp}^{3}$ 杂化, 苯基和苯并噻吩不在同一个平 面. (3)与对应苯并噻吩比较, 键长发生了变化, $\mathrm{C}(2)$ $\mathrm{C}(3)$ 的键长为 $1.308 \AA$, 比苯并噻吩的 $\mathrm{C}(2)-\mathrm{C}(3)$ 的键长 $(1.352 \AA)$ 短 ${ }^{[28]}$, 介于苯乙烯双键 $(1.30 \sim 1.37 \AA)$ 之间. 由 此可知, 硫原子苯基化后, 噻吩环的结构及立体构造发 生了较大的变化, 苯并噻吩盐噻吩环的芳香性已经破 坏, 它的 $C(2)-C(3)$ 键具有了烯键的性质 ${ }^{[19 a]}$.

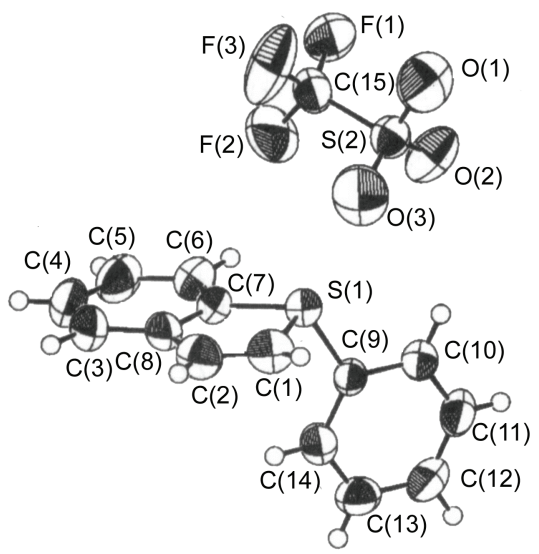

图 $1 S$-苯基苯并噻吩盐 $\mathbf{5 a}$ 的晶体结构

Figure 1 Crystal structure of $S$-phenylbenzothiophenium salt 5a

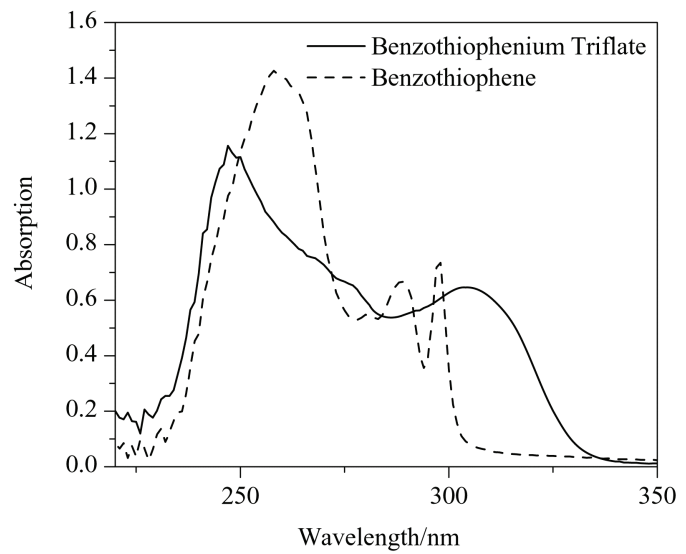

图 $2 \mathrm{~S}$-苯基苯并噻吩盐 $\mathbf{5 a}$ 和苯并噻吩的紫外吸收光谱图

Figure 2 Spectrum of the product $\mathbf{5 a}$ and benzothiophene

另外, 从苯并噻吩、 $S$-苯基苯并噻吩盐 $\mathbf{5 a}$ 的紫外吸 收光谱(图 2)可知: 从苯并噻吩到苯并噻吩盐, 其深色 位移和增色效应均消失. 这是由于 $5 \mathrm{a}$ 中硫原子上不再 存在孤电子对, 即无孤电子对与苯环及噻吩环的双键共 轭，硫原子阻止了其间的共轭作用，即具有隔离效应， 因而破坏了噻吩环闭合的共轭体系. 另外, 苯并噻吩成 盐后, 硫原子上带了部分正电荷, 表现出很强的吸电子
性, 为进行 $[4+2]$ 环化反应提供了有利条件. 由此可判 断，噻吩环上的碳碳双键的活性被提高，可以明显地表 现出烯烃的性质.

\section{S-苯基苯并噻吩盐的反应}

\section{1 与双烯的[4+2] Diels-Alder 反应}

\section{$3.1 .15 \mathbf{a}$ 与环戊二烯的环加成反应}

1999 年, Kitamura 等 ${ }^{[29]}$ 第一次报到了 $\mathbf{5 a}$ 与环戊二 烯在 $\mathrm{CH}_{2} \mathrm{Cl}_{2}$ 中反应得到产率为 $96 \%$ 的环加成产物 $\mathbf{6 a}$ (Eq. 4).

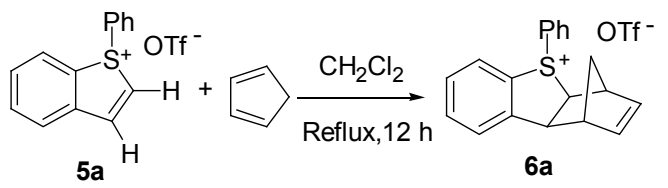

从化合物 $\mathbf{6 a}$ 的 NOE 测定结果(图 3)及 $\mathrm{X}$ 射线单晶 衍射的解析结果(图 4)表明: $\mathbf{6 a}$ 中的苯基是在噻吩环的 上面，环戊二烯组分在噻吩环的下面，6a 是内向型 $($ endo) 环加成产物. 即化合物 $\mathbf{5 a}$ 与环戊二烯反应选择性 的生成了环加成产物.

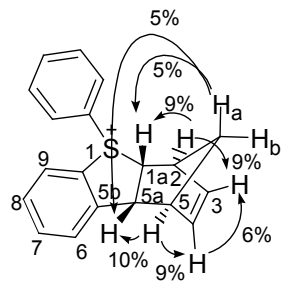

图 3 化合物 6a 的主要 $\mathrm{NOE}$ 相关

Figure 3 NOE relation in the adduct $6 \mathbf{6 a}$

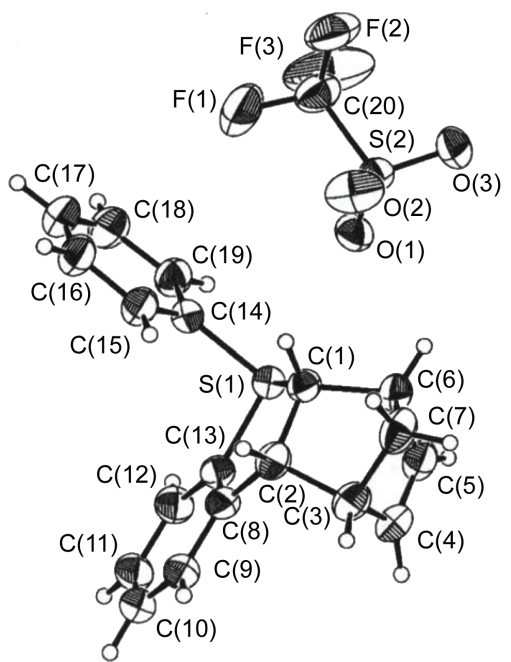

图 $46 \mathrm{a}$ 的晶体结构

Figure 4 Crystal structure of $6 a$ 
当噻吩环上带有不同取代基时, 反应在 $\mathrm{CH}_{2} \mathrm{Cl}_{2}$ 中 没有进行. 当溶剂为 $\mathrm{MeCN}$, 反应温度为 $60{ }^{\circ} \mathrm{C}$ 时, 以较 高的产率得到了对应的环化产物(Eq. 5). 化合物 $\mathbf{5}$ 的 C-2 和 C-3 位上的取代基对环加成反应有阻碍作用, 尤 其是 C-2 位的取代基对环加成反应有强的空间位阻效 应，当 C-2 位、C-3 位有苯基取代基时，没有得到对应的 产物.

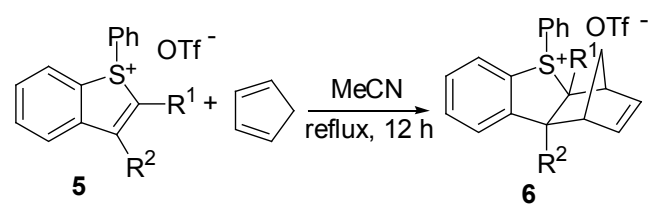

$$
\begin{aligned}
& \text { 6b: } R^{1}=M e, R^{2}=H \quad 73 \% \\
& \text { 6c: } R^{1}=H, R^{2}=\text { Me } 22 \% \\
& \text { 6d: } R^{1}=P h, R^{2}=H \quad 0 \% \\
& \text { 6e: } R^{1}=H, R^{2}=P h \quad 80 \% \\
& \text { 6h: } R^{1}=M e, R^{2}=\text { Me } 0 \% \\
& \text { 6i: } R^{1}=P h, R^{2}=\begin{array}{ll}
\text { Ph } & 0 \%
\end{array}
\end{aligned}
$$

\section{1 .2 与 1,3 -二茶基异苯并呋喃反应}

将环戊二烯替换成 1,3-二苯基异苯并呋喃与 $S$-苯基 苯并噻吩盐 $\mathbf{5}$ 进行反应时, 得到了较高产率的对应的环 加成产物 $7 \mathbf{a} \sim 7 \mathbf{c}$. 而当噻吩环上有取代基时, 特别是 C-2 位上有苯取代基时，与硫原子上的苯基相互排斥， 立体阻碍较强, 环加成反应不能够顺利进行(Eq. 6).

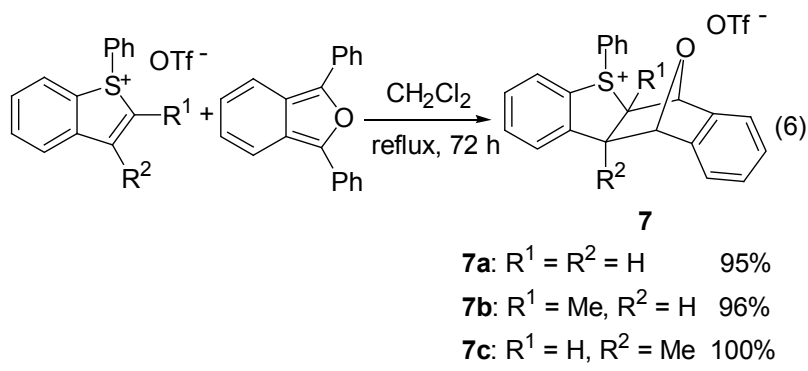

\section{2 加热反应及其机理}

将 $S$-苯基苯并噻吩盐 $\mathbf{5}$ 在固体状态下, $180{ }^{\circ} \mathrm{C}$ 加热 $30 \mathrm{~min}$ 后, 经柱色谱分离后得到的产物是硫原子上的苯 基发生迁移的取代苯并噻吩 ${ }^{[30]}$. 无取代基的情况下, $S$ 苯基选择性的迁移到 C-2 位; 当 C-2 上有取代基时, $S$ 苯基选择性地迁移到 C-3 位(Eqs. 7, 8).

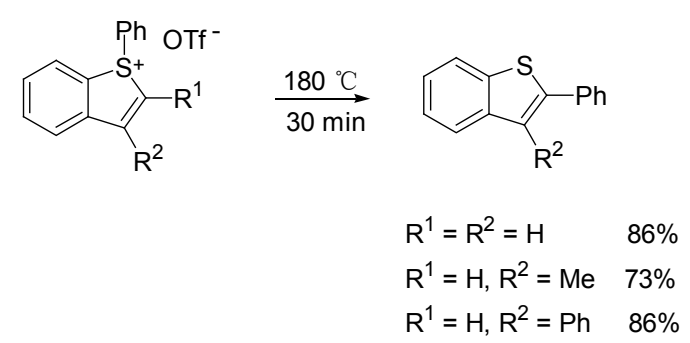<smiles></smiles><smiles>[R]c1sc2ccccc2c1-c1ccccc1</smiles>

$\mathrm{R}^{1}=\mathrm{Me}, \mathrm{R}^{2}=\mathrm{H} \quad 78 \%$

$\mathrm{R}^{1}=\mathrm{Ph}, \mathrm{R}^{2}=\mathrm{H} \quad 74 \%$

另一方面，当 C-2 位上有对甲基苯基( Tol)时，在 $180{ }^{\circ} \mathrm{C}$ 下加热 $1 \mathrm{~h}$ 后, 得到了 $S$-苯基迁移到 C-3 位的产 物 $\mathbf{a}$ 和 $S$-苯基迁移到 C-2 位、 $\mathrm{Tol}$ 迁移到 $\mathrm{C}-3$ 位的产物

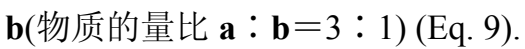

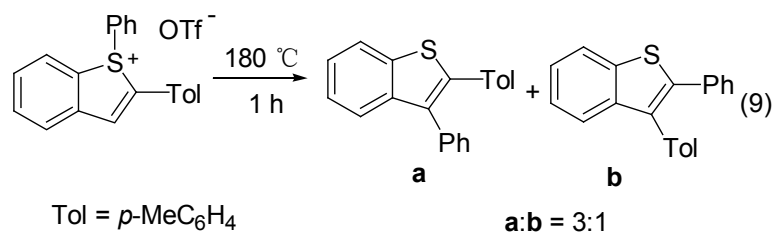

由以上实验结果可以推测： $S$-苯基苯并噻吩盐的加 热分解反应机理如 Scheme 4 所示, 硫原子上的苯基首 先进行 $[1,5]-\mathrm{Ph}$ 迁移, 当 C-2 位、C-3 位无取代时, 迅速 发生脱氢反应，生成稳定的 2-苯基苯并噻吩; 当 C-2 位 上有取代基时苯基首先进行一次 $[1,5]-\mathrm{Ph}$ 迁移到 C-2 位, 生成中间体 I. 当两个基团不一致时, 将与 C-2 位原有的 取代基发生迁移竞争，最后生成两种较稳定的迁移产 物. 例如，当 C-2 位有对甲基苯基(Tol)时，苯基的迁移 优先于对甲苯基, 得到了发生两次 [1,5]-Ph 的主产物 $\mathbf{a}$.

\section{3 光解反应及机理}

与以上加热反应相对应，将 $S$-苯基苯并噻吩盐在 $\mathrm{N}_{2}$ 保护下 $\mathrm{CH}_{2} \mathrm{Cl}_{2}$ 溶剂中, 用 $\mathrm{Hg}$ 灯 $(100 \mathrm{~W})$ 光照 $1 \mathrm{~h}$ 后, 得到了不同的光解产物 ${ }^{[31]}$ (Eq. 10).

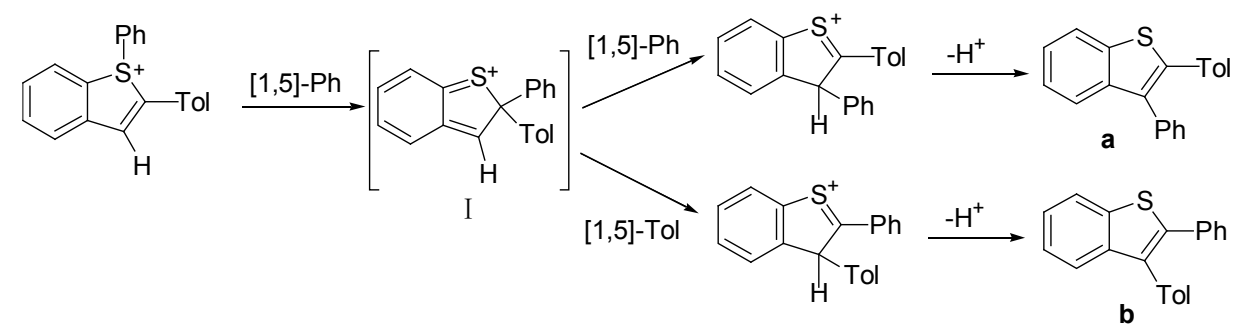

Scheme 4 

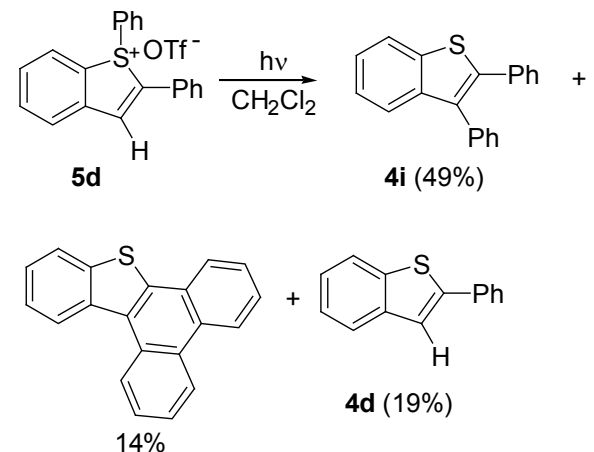

在同样的条件下，化合物 $\mathbf{5 c}$ 光解成产率为 $36 \%$ 的 $4 \mathrm{~g}$ 和 $2 \%$ 的 $4 \mathrm{c}$, 而化合物 $5 \mathrm{~b}$ 的光解反应没有得到苯基 迁移的产物, 得到了产率为 $66 \%$ 的单一化合物 $4 \mathrm{~b}$ (Eqs. $11,12)$.

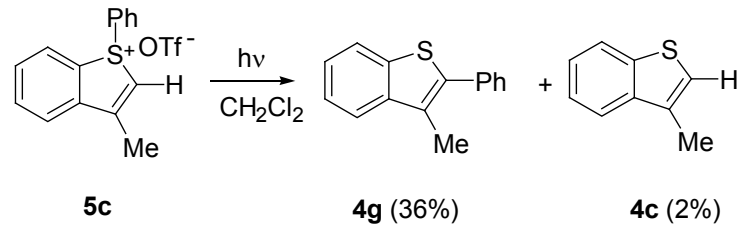

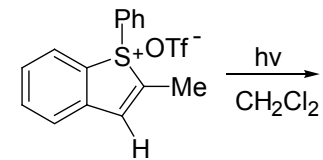

$5 b$
$4 b(66 \%)$<smiles>Cc1cc2ccccc2s1</smiles>

在 $S$-苯基苯并噻吩盐的加热分解中, $\mathrm{Ph}-\mathrm{S}$ 键断裂, 苯基发生了选择性的迁移. 在光分解反应中, 不仅有苯 基发生迁移的产物, 而且还有随着 $\mathrm{Ph}-\mathrm{S}$ 的断裂脱苯基 化的苯并噻吩产物. 由此可知: 在光解反应中, 与加热 分解的情况不同, 没有进行 $\mathrm{S}-\mathrm{C}$ 键的断裂, 而是发生 了 $\mathrm{Ph}-\mathrm{S}$ 键的均裂, 产生的苯基自由基和苯并噻吩自由 基在溶剂效应中进行再结合(Scheme 5).

\section{4 苯并噻吩盐在亲核试剂中发生开环反应}

在 $S$-苯并噻吩盐与亲核试剂的反应中, 是否发生取 代或者消除反应取决于 C-2 位、C-3 位上的取代基 ${ }^{[32]} .8$
与甲醇钠在室温下反应 $12 \mathrm{~h}$ 后，得到了产率为 $73 \%$ 的开 环产物 11，在室温下 9 和 10 不与甲醇钠反应，当在甲醇 中回流 $24 \mathrm{~h}$ 后，得到了产率分别为 100\%的甲氧基取代 的产物 12 和 13.

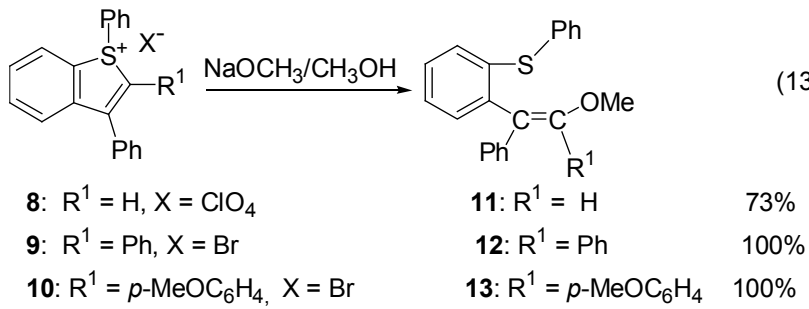

当 C-3 位上有苯基, C-2 位上有甲基或丙基取代基 时，苯并噻吩盐与甲醇钠反应没有生成有甲氧基取代的 开环产物, 得到了相应的消除产物. 例如化合物 14 与甲 醇钠在室温下反应 $12 \mathrm{~h}$, 得到了产率为 $89 \%$ 的联二烯 16, 在相同条件下，化合物 15 与甲醇钠反应得到了产率 为 $100 \%$ 的二烯 17 .

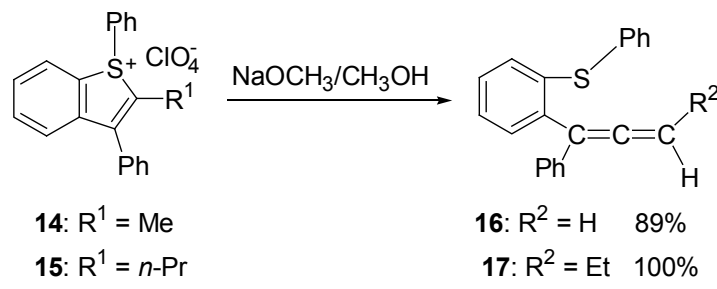

C-3 位无取代基时，苯并噻吩盐与甲醇钠反应生成 了开环产物, 例如化合物 18,19 和 $5 \mathrm{a}$ 与甲醇钠在室温反 应 $12 \mathrm{~h}$ 后，得到了产率几乎为 $100 \%$ 的 $\mathbf{2 0 , 2 1}$ 和 22 的炔 化合物。

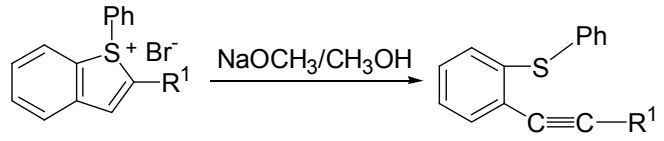

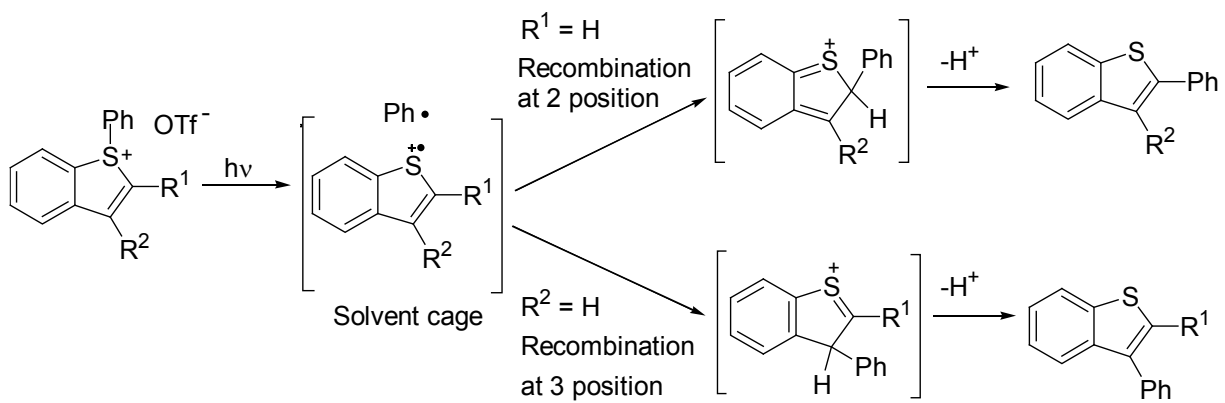

Scheme 5 
<smiles>Oc1ccccc1</smiles>

5a<smiles>C#Cc1ccccc1SP</smiles>

22
由 $S$-苯基苯并噻吩为原料进行 $[4+2]$ 反应后得到的 环状加成产物 6 和 7 室温下在甲醇钠的醇溶液中较稳定, 当反应温度升高到 $50{ }^{\circ} \mathrm{C}, 6 \mathbf{a}$ 开环生成了产率为 $86 \%$ 的 23. 在同样的条件下, 7a 开环生成了产率为 $96 \%$ 的 24 . 但是有取代基的环状加成产物 $6 \mathrm{c}$ 没有发生开环反应, 可能是多环结构阻碍了亲核试剂甲醇钠的对硫进攻 ${ }^{[29 b]}$.

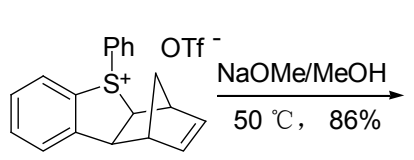

6a

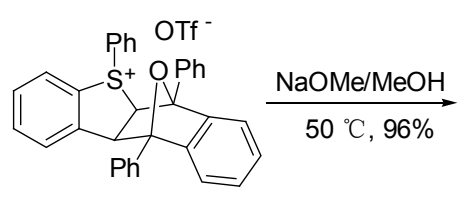

$7 a$

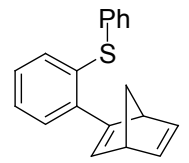

23<smiles>C1=CC2(c3ccccc3)OC1(c1ccccc1)CC2c1ccccc1Sc1ccccc1</smiles>

24

\section{4 结论展望}

用醋酸铜作催化剂, 在无溶剂的条件下, 由苯并噻 吩与高碘盐反应，可以一步合成在有机合成上被认为是 困难的 $S$-苯并噻吩盐, 为苯并噻吩盐的合成提供了一个 非常简便而有效的绿色型方法. 从理论的角度和 $[4+2]$ Diels-Alder 反应的实验结果可以证明苯并噻吩成盐以 后原有的芳香性发生变化, 噻吩环上的 $\mathrm{C}=\mathrm{C}$ 双键表现 出明显的烯烃的性质, 能够合成系列新型的苯并噻吩衍 生物, 不仅为天然化合物以及农药、医药品的合成提供 了原料, 而且为新药的开发提供了理论依据. 目前有关 苯并 $[b]$ 噻吩盐的实际应用例子较少. 三芳基锍鎓盐可 用作阳离子光引发剂、相转移催化剂、光生酸剂、阳离 子苂光探针以及生物传感器. 以苯并噻吩为骨架的锍鎓 盐在波长为 $270 \sim 340 \mathrm{~nm}$ 之间有较强的紫外吸收, 同时 在光照和加热条件下硫原子上的芳基能发生分子内迁 移并放出强酸, 有望作为光引发剂在功能性材料、紫外 光固化领域得到应用。

\section{References}

[1] Liu, H.; Bolton, J. L.; Thatcher, G. R. Chem. Res. Toxicol. 2006, 19, 779.

[2] Li, J.-Y.; Zhou, X.-R.; Zhao, D.-F. Chemistry 2005, 68, 399 (in Chinese).

(李建源, 周新锐, 赵德丰, 化学通报, 2005, 68, 399.)

[3] (a) Iwasaki, T.; Kohinata, Y.; Nishide, H. Org. Lett. 2005, 7, 755. (b) Zhang, B.-X.; Shi, G.-S.; Wang, Q.; Kang, J.-L. J. Shanxi Univ. 2011, 34, 90 (in Chinese).

(张变香, 石高升, 王琼, 杨祺, 康婧玲, 山西大学学报, 2011, 34, 90.)

[4] Chen, Q.; Ngui, J. S. Chem. Res. Toxicol. 2002, 15, 907.

[5] Xiong, F.; Prince; Chen, C.; Yang, X.-P. J. East China Normal Univ. 2001, (3), 109 (in Chinese).

(熊飞, Prince, 陈春, 杨琍苹, 华东师范大学学报, 2001, (3), 109.)

[6] Takimiya, K.; Ebata, H.; Sakamoto, K.; Izawa, T.; Otsubo, T.; Kunugi, Y. J. Am. Chem. Soc. 2006, 128, 12604.

[7] Britton, T. C.; Spinazze, P. G.; Hipskind, P. A.; Zinuncrman, D. M.; Zardnrnayeh, H.; Schober, D. A.; Gghlert, D. R.; Bruns, R. F. Bioorg. Med. Chem. Lett. 1999, 9, 475.

[8] Efange, S. M. N.; Mash, D. C.; Khare, A. B.; Ouyang, Q. J. Med. Chem. 1998, 41, 4486.

[9] Wallace, O. B.; Bryant, H. U.; Shetler, P. K.; Adrian, M. D.; Geiser, A. G. Bioorg. Med. Chem. Lett. 2004, 14, 5103.

[10] Jacobi, P. A.; Touchette, K. M.; Selnick, H. G. J. Org. Chem. 1992, $57,6305$.

[11] Balley, J.; Cummins, E. W. J. Am. Chem. Soc. 1954, 76, 1936.

[12] Raasch, M. S. J. Org. Chem. 1980, 45, 856.

[13] Crivello, J. V.; Lam, J. H. W. J. Org. Chem. 1978, 43, 3055.

[14] Wang, M.-L.; Wang, W.-G.; Han, Y.-L. Imaging Sci. Photochem. 2008, 26, 88 (in Chinese).

(王美丽, 王文广, 韩元利, 影像科学与光化学, 2008, 26, 88.)

[15] Wang, W.-J. J. Envir. Manage. College China 2005, 15, 55 (in Chinese). (王文君, 中国环境管理干部学院学报, 2005, 15, 55.)

[16] (a) Acheeon, R. M.; Harrison, D. R. J. Chem. Soc., Chem. Commun. 1969, 724.

(b) Acheeon, R. M.; Harrison, D. R. J. Chem. Soc., Chem. Commun. 1976, 612.

[17] Shiraishi, Y.; Taki, Y.; Hirai, T.; Komasawa, I. Ind. Eng. Chem. Res. 2001, 40, 1213.

[18] Brummond, K. M.; Chen, D. Org. Lett. 2005, 7, 3473.

[19] Green, T. K.; Whitley, P.; Wu, K. N.; Lloyd, W. J.; Gan, G. Z. Energy Fuels 1994, 8, 244.

[20] Brumlik, G. C.; Kosak, A. I.; Pitcher, R. J. Am. Chem. Soc. 1964, 86,5360 .

[21] (a) Kitamura, T.; Kawasato, H.; Kobayashi, S.; Taniguchi, H. Chem. Lett. 1986, 38, 399.

(b) Kitamura, T.; Soda, S.; Kawasato, H.; Taniguchi, H.; Shiro, M. Tetrahedron 1993, 49, 5055.

[22] (a) Kitamura, T.; Takachi, T.; Kawasato, H.; Kobayashi, S.; Taniguchi, H. Tetrahedron Lett. 1989, 30, 7445.

(b) Kitamura, T.; Takachi, T.; Miyaji, M.; Kawasato, H.; Taniguchi, H. J. Chem. Soc., Perkin Trans. 1 1994, 1907.

[23] (a) Kitamura, T.; Yamane, M.; Furuki, R.; Taniguchi, H.; Shiro, M. Chem. Lett. 1993, 1703.

(b) Kitamura, T.; Yamane, M.; Zhang, B. X.; Fujiwra, Y. Bull. Chem. Soc. Jpn. 1998, 71, 1215.

[24] Beringer, F. M.; Gindler, E. M.; Rapoport, M.; Tayler, R. J. J. Am. Chem. Soc. 1959, 81, 351.

[25] Caserio, M. C.; Glusker, D. L.; Roberts, J. D. J. Am. Chem. Soc. 1959, $81,336$.

[26] Lockhart, T. P. J. Am. Chem. Soc. 1983, 105, 1940.

[27] Phipps, R. J.; Grimster, N. P.; Gaunt, M. J. J. Am. Chem. Soc. 2008, 130,8172 .

[28] Dewar, M. J. S.; Trinajsti'c, N. J. Am. Chem. Soc. 1970, 92, 1453.

[29] (a) Kitamura, T.; Zhang, B. X.; Fujiwara, Y.; Shiro, M. Org. Lett. 1999, $1,257$. 
(b) Kitamura, T.; Zhang, B. X.; Fujiwara, Y. J. Org. Chem. 2003, 68,731 .

(c) Zhang, B.-X.; Wang, Q. J. Shanxi Univ. 2008, 31, 80 (in Chinese).

(张变香, 王琼, 山西大学学报, 2008, 31, 80.)

[30] Kitamura, T.; Zhang, B. X.; Fujiwara, Y. Tetrahedron Lett. 2002,
43, 2239.

[31] Kitamura, T.; Morizane, K.; Taniguchi, H.; Fujiwara, Y. Tetrahedron Lett. 1997, 38, 5157.

[32] Kitamura, T.; Yamane, M.; Soda, S.; Taniguchi, H. J. Chem. Soc., Chem. Commun. 1995, 1375. 